\title{
AC Dielectrophoresis Using Elliptic Electrode Geometry
}

\author{
S. M. Rezaul Hasan and Aanan Khurma \\ Center for Research in Analog and VLSI Microsystem Design (CRAVE), School of Engineering and Advanced Technology (SEAT), \\ Massey University, Albany, Auckland 0632, New Zealand
}

Correspondence should be addressed to S. M. Rezaul Hasan, hasanmic@massey.ac.nz

Received 6 December 2010; Revised 27 March 2011; Accepted 27 March 2011

Academic Editor: K. Kalantar-Zadeh

Copyright ( $) 2011$ S. M. R. Hasan and A. Khurma. This is an open access article distributed under the Creative Commons Attribution License, which permits unrestricted use, distribution, and reproduction in any medium, provided the original work is properly cited.

\begin{abstract}
This paper presents negative AC dielectrophoretic investigations using elliptic electrode geometry. Simulations of the electric field gradient variation using various ratios of the semimajor and the semiminor axis were carried out to determine the optimum elliptic geometry for the dielectrophoretic electrokinetics of specimen in an assay with laminar (low Reynolds number) fluid flow. Experimental setup of the elliptic electrode assembly using PCB fabrication and electrokinetic accumulation of specimen in a dielectrophoretic cage is also being reported. Using an actuating signal between $1 \mathrm{kHz}$ and $1 \mathrm{MHz}$, successful trapping of $45 \mu \mathrm{m}$ polystyrene beads suspended in distilled water was demonstrated due to negative dielectrophoresis near $100 \mathrm{kHz}$ using the novel elliptic electrode.
\end{abstract}

\section{Introduction}

Advances in bioelectronic miniaturization is bringing forward the dream of creating a true lab-on-a-chip (LOAC) closer to reality where sensing, processing, and actuation functions are integrated on the same platform. The numerous areas of application of LOAC $[1,2]$ technology include diagnostics, therapeutics, ecological monitoring, and drug discovery. An LOAC with fluidic microchannels for electronic manipulation of particles/molecular species can act as a point-of-care device driving down health care costs. In addition to providing portability, a more technologically specific advantage of miniaturization is the possibility of manipulating fluids and bioparticles using electric fields and temperature gradients, which is intangible under normal macroscopic circumstances. Such devices are being progressively researched, designed, and fabricated for providing rapid sensing, separation, collection, preparation, and identification of biological specimen. For example, the analysis of proteins which easily adsorb, degrade, or aggregate as compared to nucleic acids, is faster, easier, and less costly to handle on an LOAC compared to other techniques such as gel electrophoresis. The application of the phenomenon of dielectrophoresis (DEP) in manipulating uncharged diel- ectric biological species (molecules, protein, DNA [3], etc.) suspended in fluidic channels in the presence of AC electrical field gradient has attracted significant attention in the fabrication of LOAC devices [4]. With the emergence of microtechnology, it has became possible to fabricate electrodes on the microscale allowing generation of the large gradients in electric field intensity, which are necessary for the DEP-force to reach a magnitude suitable for electrokinetic [5] translation of microscopic particles/species. A major advantage is that the particle does not have to be chemically tagged and translation is achieved by creating a dipole on a neutral particle. Depending on the relative dielectric permittivity $(\varepsilon)$ of the particle with respect to the suspending fluid, particles/species can be transported and trapped in a region in the direction of the electric field gradient (positive DEP or pDEP) [6] or in a region in the direction opposite to it (negative DEP or $\mathrm{nDEP}$ ) [6]. A subset of the DEP translation technique is the traveling wave dielectrophoresis (twDEP) [7] which is achieved using DEP electrode array with quadrature sinusoids covering one phasor rotation. The manipulation of dielectric particles using AC electric fields is, however, not restricted to translational displacements. Electrorotational torque (ROT) has also been achieved on a single specimen using a rotating AC electric field [8]. 
DEP manipulation of species can be varied based on the type of electrode geometry [9]. The different categories of electrode geometry reported so far $[10,11]$ include parabolic geometry, castellated (with possible interdigitation [12]) electrode geometry, and array geometries using parallel rectangular tracks or its triangular variations. Variation of the electrode geometry changes the pattern of the non-uniform (nonhomogenous) electric field resulting in significant effects on the electrokinetic motion of species in suspending fluids within the DEP device. Variations in species behavior within the DEP device has advantages in sample preparation in an LOAC application. In this paper, investigations into elliptic electrode design for DEP for specimen/particle separation and trapping are being reported. The aim of this electrode design was to create distinct bands of large electric field gradients for controlling species movement and trapping the desired species/particles using nDEP in a chamber with laminar (low Reynolds number) fluid flow. Cells traveling in one laminar flow can be guided into another by using nDEP [13].

\section{Dielectrophoresis Fundamentals}

\subsection{Polarization of Neutral Dielectric Sphere by a Static Homogeneous Field}

It is well known that neutral dielectric particles such as biocells are polarized under the influence of an electric field. A spherical dielectric particle placed in a homogeneous electric field $E_{\text {hom }}$ is polarized in such a manner that the electric field $E_{\text {pol }}$ generated by this polarization corresponds exactly to the electric field due to an electric dipole of dipole moment $p$ placed at the center of the sphere. This dipole moment $p$ is given by [14]

$$
p=4 \pi \varepsilon_{m} \frac{\varepsilon_{p}-\varepsilon_{m}}{\varepsilon_{p}+2 \varepsilon_{m}} a^{3} E_{\mathrm{hom}},
$$

where, $\varepsilon_{p}$ and $\varepsilon_{m}$ are the dielectric constants of the particle/species and the surrounding media/fluid, respectively, and $a$ is the radius of the particle. The fraction $\left(\varepsilon_{p}-\varepsilon_{m}\right) /\left(\varepsilon_{p}+\right.$ $2 \varepsilon_{m}$ ) dictates the direction of $p$ with respect to $E_{\text {hom }}$ and is known as the Clausius-Mossotti factor, $K\left(\varepsilon_{p}, \varepsilon_{m}\right)$.

2.2. Dielectrophoretic Force on a Small Particle. The simple deduction (1) facilitates the treatment of a particle/bio-cell as a dipole when calculating the force exerted on the particle by the electric field. The dielectrophoretic force $F_{\mathrm{DEP}}$ on a dipole situated in an electric field $E$ is given by [14-17]

$$
F_{\mathrm{DEP}}=p \cdot \nabla E
$$

In (2) the gradient of $E$ is assumed to be over a distance $d$ with particle radius $a \ll d$, so that $E$ is largely homogenous within close vicinity of the particle. Thus, (1) and (2) can be applied simultaneously even in a non-uniform electric field with a gradient for determining the dielectrophoretic force on a suspended dipole particle. $F_{\mathrm{DEP}}$ is then given by

$$
F_{\mathrm{DEP}}=4 \pi \varepsilon_{m} \frac{\varepsilon_{p}-\varepsilon_{m}}{\varepsilon_{p}+2 \varepsilon_{m}} a^{3} E \cdot \nabla E
$$

or

$$
F_{\mathrm{DEP}}=4 \pi \varepsilon_{m} \frac{\varepsilon_{p}-\varepsilon_{m}}{\varepsilon_{p}+2 \varepsilon_{m}} a^{3} \nabla E^{2}
$$

In (4), the electric field appears only as a squared quantity indicating that the direction of $E$ has no significance with regard to the direction of the DEP force. The magnitude of the DEP force is mostly determined by the size (surface area) of the particle and the electric field gradient, while the direction of the DEP force is determined by the sign of the Clausius-Mossotti factor. If $K<0$, the particle will be repelled by regions of high field gradients which is called negative dielectrophoresis or $\mathrm{nDEP}[6]$. On the other hand, If $K>0$, the particle will be attracted towards areas of higher field gradients which is called positive dielectrophoresis or pDEP [6].

2.3. The Dielectrophoretic Force Generated by an AC Electric Field. The dielectrophoretic force subjected to a dielectric particle/species by a sinusoidal electric field of the form, $E(r, t)=E(r) e^{j \omega t}$, is given by $[15,16]$,

$$
F_{\mathrm{DEP}}(r, \omega)=2 \pi \varepsilon_{m} \frac{\widetilde{\varepsilon}_{p}-\widetilde{\varepsilon}_{m}}{\widetilde{\varepsilon}_{p}+2 \widetilde{\varepsilon}_{m}} a^{3} \nabla E_{\mathrm{rms}}^{2}(r, \omega)
$$

where the gradient is with respect to the radial distance $r, \widetilde{\varepsilon}_{p}$ and $\widetilde{\varepsilon}_{m}$ are frequency dependent complex permittivities given by $\widetilde{\varepsilon}_{p}=\varepsilon_{p}+\sigma_{p} / j \omega$ and $\widetilde{\varepsilon}_{m}=\varepsilon_{m}+\sigma_{m} / j \omega$, respectively. Also, $\sigma_{p}$ and $\sigma_{m}$ are, respectively, the conductivities of the particle and the medium. The conductivity of the particle with radius $a$ is given by

$$
\sigma_{p}=\sigma_{\text {bulk }}+2 \frac{K_{s}}{a}
$$

where $\sigma_{\text {bulk }}$ is the bulk conductivity and $K_{s}$ is the total surface conductance for the particle. Considering fabricated test particles, $\sigma_{\text {bulk }}$ is negligible for good insulators, for example, polystyrene [18], while the overall particle conductivity reduces with particle radius. $K_{s}$ for manufactured particles is composed of the stern layer and the diffuse layer formed around the particle. The value of $K_{s}$ vary widely, being approximately in the range $1-10 \mathrm{nS}$ [18]. This value is largely independent of particle radius [18], and the wide variation is mostly attributed to particle manufacturer's fabrication process and particle surface features $[18,19]$. As in the static case, the real part of the complex Clausius-Mossotti factor, $K$, that is, $\operatorname{Re}\left[\left(\widetilde{\varepsilon}_{p}-\widetilde{\varepsilon}_{m}\right) /\left(\widetilde{\varepsilon}_{p}+2 \widetilde{\varepsilon}_{m}\right)\right]$, essentially determines the sign of $F_{\mathrm{DEP}}(r, \omega)$. However, as opposed to the static case, $K$ is now frequency dependent. Particles of different conductivities and permittivities are affected by different frequency fields. Figure 1 shows the simulated variation of the real part of the $K$ factor with frequency, and, the DEP crossover frequencies for $6 \mu \mathrm{m}, 20 \mu \mathrm{m}, 30 \mu \mathrm{m}$, and $45 \mu \mathrm{m}$ particles (particle size standards from Sigma-Aldrich [20]) using an approximate median $K_{s}$ value of $5 \mathrm{nS}$ based on the discussions in [18]. Dielectric properties of distilled water was used for the simulations as the suspending media. Actual DEP force will also depend on the electrode geometry. 


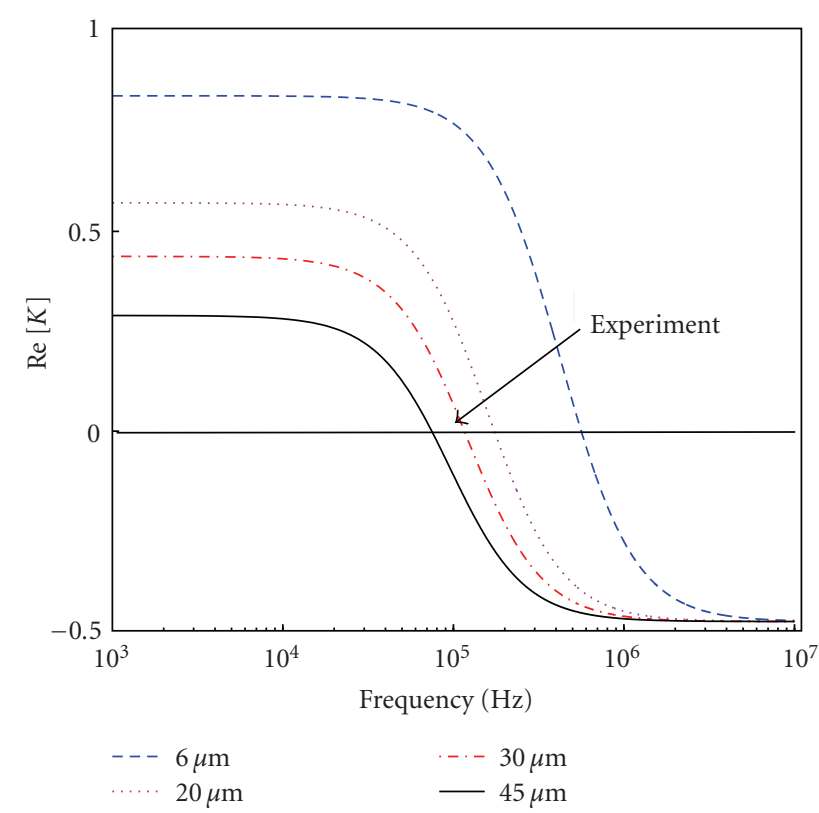

Figure 1: Approximate variation of $\operatorname{Re}[K(\omega)]$ with frequency for polystyrene particles of various sizes.

In addition, small variations in the conductivity of the medium can greatly affect the observed crossover frequency [21]. Considering the effect of viscous friction on dragged particles, the dielectrophoretic mobility can be defined [5] as

$$
\mu_{\mathrm{DEP}}=\frac{\left(\varepsilon_{m} \operatorname{Re}[K(\omega)] a^{2}\right)}{(6 \eta)},
$$

where $\eta$ is the medium viscosity. When particles move at constant speed, the time required to cover the small distance $\Delta d$ is given by

$$
\tau_{\mathrm{DEP}}=\frac{\Delta d}{\left(\mu_{\mathrm{DEP}} \Delta E_{\mathrm{rms}}^{2}(r, \omega)\right)} .
$$

Equations (7) and (8) indicate the effect of scaling the dimensions of the electrode and the suspended particles/species.

\section{Elliptic Electrodes for AC Dielectrophoresis}

\subsection{Design, Simulation, and Experimental Results}

The construction of the proposed elliptic electrodes for dielectrophoretic actuation is quite straight-forward. It consists of an elliptic chamber with conducting sidewalls and two thin conductors placed at the foci of the ellipse. A microchannel is provided for passage of particles in a laminar flow. Figure 2 shows a typical design of the elliptic electrode in PCB where all the distances are shown in $\mu \mathrm{m}$. Here, the semiminor axis is $3500 \mu \mathrm{m}$ while the semimajor axis is $4000 \mu \mathrm{m}$. The microchannel provided can be used for remov-al of collected/separated particles. Cadsoft Eagle PCB layout editor was used for the electrode design while PROTOMAT S62 was used for machining. Figure 3 shows a scheme for designing an array of elliptic electrodes of different sizes on the same

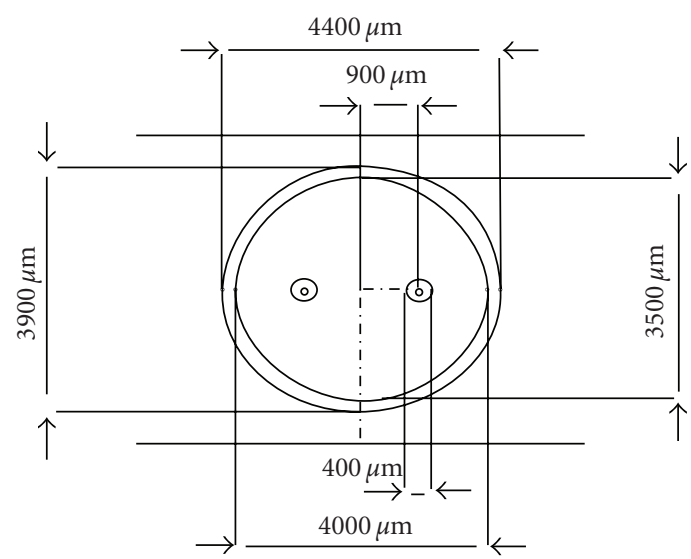

(a)

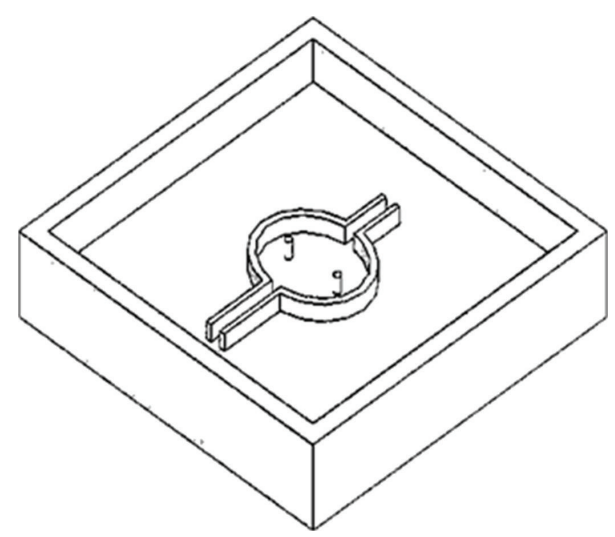

(b)

Figure 2: A design scheme for an elliptic electrode using Protomat S62 PCB prototyping, (a) design sketch with dimensions, and (b) a $3 \mathrm{D}$ solid model.

PCB-substrate with a constant semimajor axis, ax $1=4 \mathrm{~mm}$ and the semiminor axis, ax2 varying between $3.8 \mathrm{~mm}$ and $1.2 \mathrm{~mm}$. The material used for the electrodes is copper. Since the device was fabricated using PCB based miniaturization techniques, there was a constraint on the smallest possible dimension which is around $100 \mu \mathrm{m}$ for theoretical purposes and around $150-200 \mu \mathrm{m}$ for all practical purposes. The smallest device dimension used here was $200 \mu \mathrm{m}$, and, thus, the device was free from PCB fabrication imperfections. The two inner electrodes were placed very close to the actual foci of the ellipse and the margin is practically negligible. The working principle of the electrode arrangement is AC dielectrophoresis where the AC electric fields would cause movement of uncharged particles. To achieve this, the two inner electrodes were excited using an in-phase sinusoidal stimulus while the outer elliptic electrodes was excited using a counterphase sinusoidal stimulus. Before the PCB prototyping, electric field simulations were carried out to visualize the electric field produced in the elliptic electrode assembly due to such a combination of excitations. The simulations were performed using MATLAB-7.0 and confirmed using Ansoft 2D electromagnetic field simulator MAXWELL. Figures 4(a)-4(e) show the results of the electric 
Central electrode connections

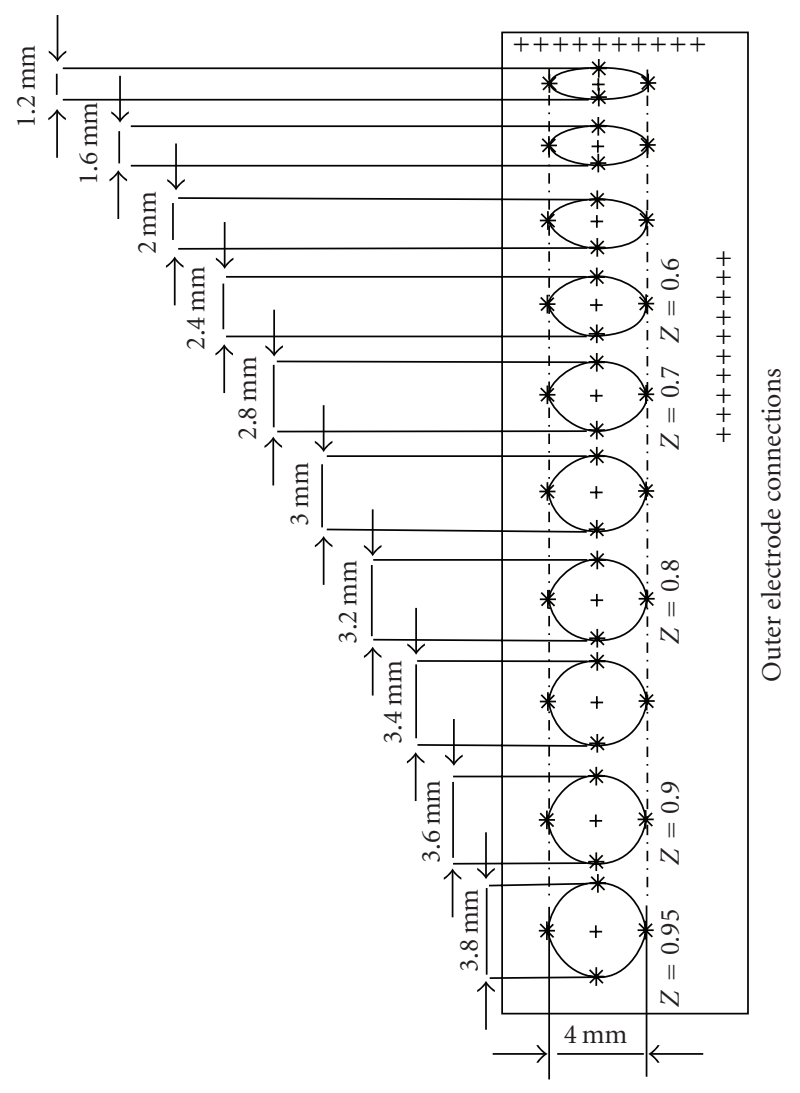

FIGURE 3: A scheme for the construction of an array of elliptic electrodes on the same substrate.

field simulations for different values of the axis ratio, " $Z$ " = "ax2/ax1". In Figure 4(a), for $Z=0.6$, the region of electric field minima is not that prominent. A large gradient exists in the region close to the elliptic electrodes while the rate of change of electric field intensity between the two electrodes at the foci is imperceptible. Hence, $Z=0.6$ is not a good choice of elliptic electrode dimensions. Figures 4 (b) to 4(e) shows improvements in the electric field gradient for negative DEP force that can accumulate particles near the center between the two inner foci electrodes. As microparticles often tend to stick to metal electrodes with voltaic connections if they are allowed to contact them, it is highly advantageous [22] to repel them from such areas through negative DEP. This is generally assured by using a suspending fluid with higher polarizibility compared to the suspended particles/species. Experimentally, it requires an AC dielectrophoretic excitation frequency above the crossover frequency based on the $K$-factor simulations depicted in the Figure 1.

Following the electric field simulations, the negative DEP device was thus constructed containing 8 elliptic electrodes on a single substrate, each with a different $Z$ value. This allowed obtaining the optimum $Z$ value from experimental results with samples. Figure 5(a) is a top view of the fabricated electrode assembly showing voltaic connections to the outer electrodes, while Figure 5(b) shows the bottom (backside) of the fabricated electrode assembly depicting the voltaic connections to the inner electrodes. Microchannels (inlet and outlet) for laminar fluid flow are provided at the edges of the electrode along the semiminor axis. The stimuli applied to the electrodes were obtained using a balanced op-amp circuit. The wide bandwidth quad-JFET LF-347 op-amp (supply-voltage $= \pm 18 \mathrm{~V}$ and output voltage swing $= \pm 15 \mathrm{~V}$ ) was used for this purpose. The input to the circuit was provided using a standard laboratory function generator. The circuit was simulated on National Instrument Multisim as shown in Figure 6. The device was tested using particles and suspending medium of different conductivities and permittivities. The particles were added to the suspending media in a standard test tube, and the suspension was then dispensed into the electrode cavity using a dropper. However, in the test frequency range for example, $1 \mathrm{kHz}$ to $1 \mathrm{MHz}$, successful results were only obtained for combination of polystyrene particles and distilled water. Distilled water has conductivity of $4-10 \mu \mathrm{S} / \mathrm{cm}$, which is much higher than pure water $(0.055 \mu \mathrm{S} / \mathrm{cm})$, and relative permittivity $\left(\varepsilon_{\text {rel }}\right)$ of $78[19,23]$. The conductivity increases in the presence of aerial carbon dioxide $\left(\mathrm{CO}_{2}\right)$ in close contact with the surface. Polystyrene particles, on the other hand, have widely variable conductivity as per (6) (discussion in Section 2), and relative permittivity of 2.4-2.7. The permittivities are relative to the permittivity of free space which is $8.85 * 10^{-12} \mathrm{Farad} / \mathrm{m}$. This combination of polystyrene beads and distilled water thus 


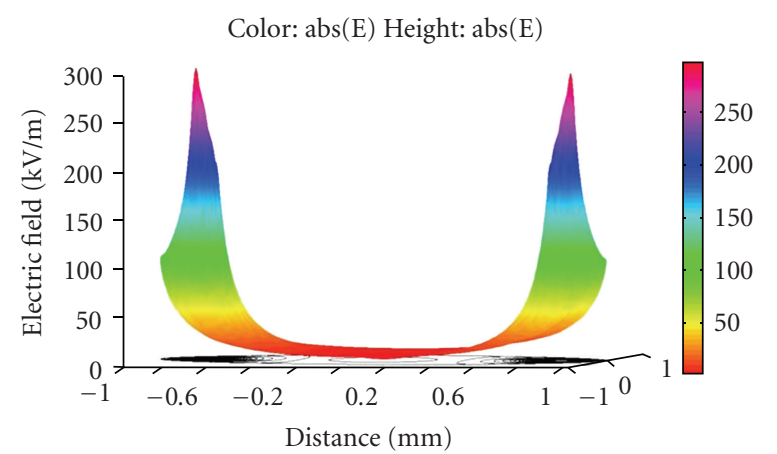

(a)

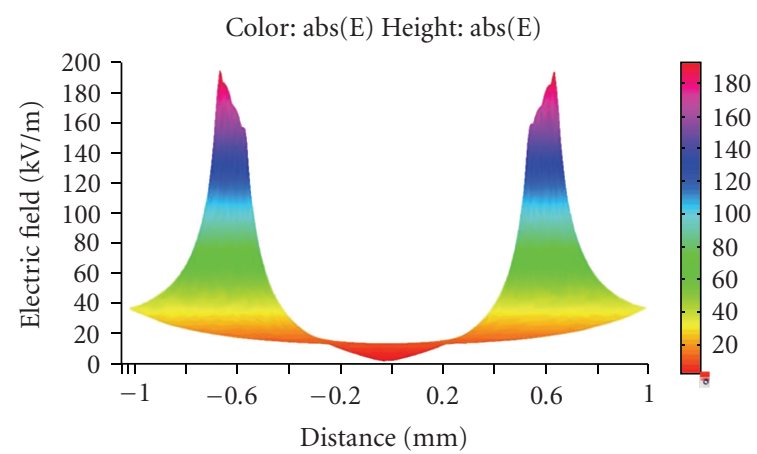

(c)

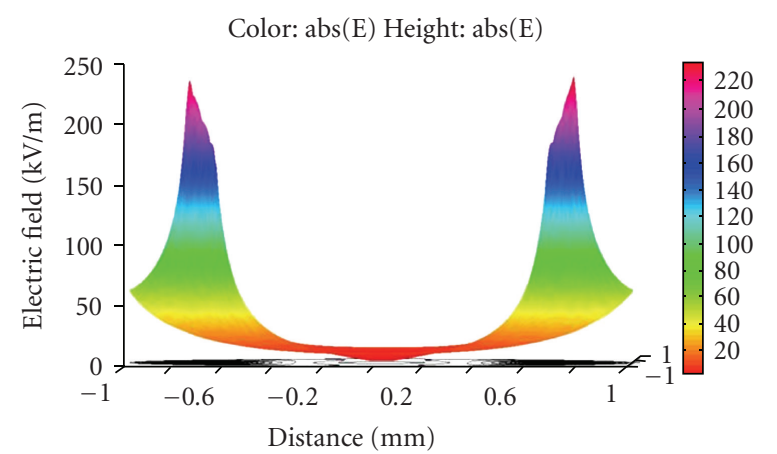

(b)

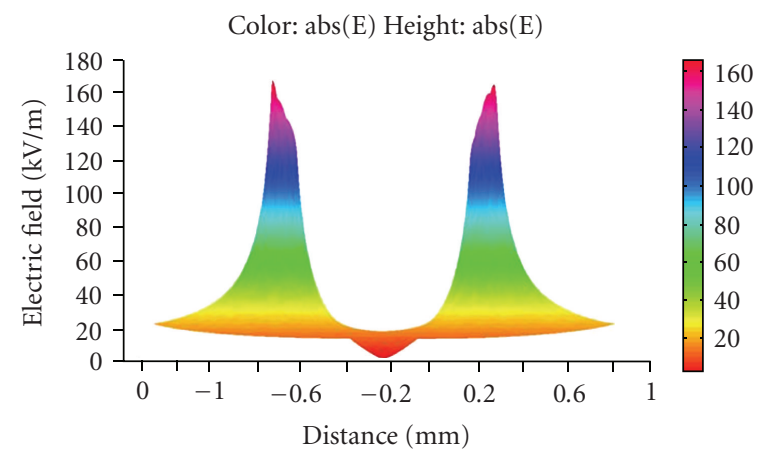

(d)

Color: abs(E) Height: abs(E)

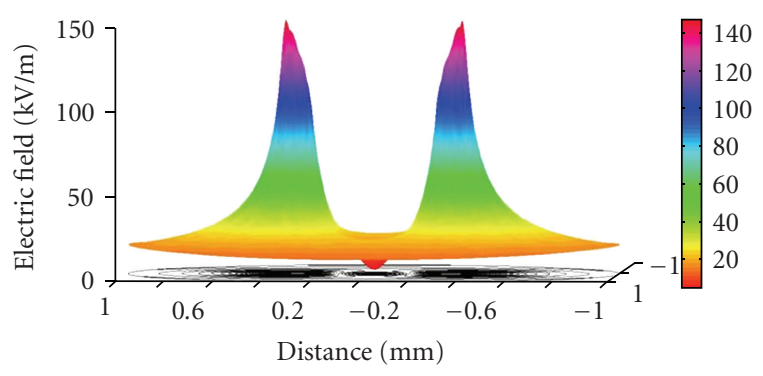

(e)

Figure 4: Electric field pattern for (a) $Z=0.6$, (b) $Z=0.7$, (c) $Z=0.8$, (d) $Z=0.9$, and (e) $Z=0.95$.

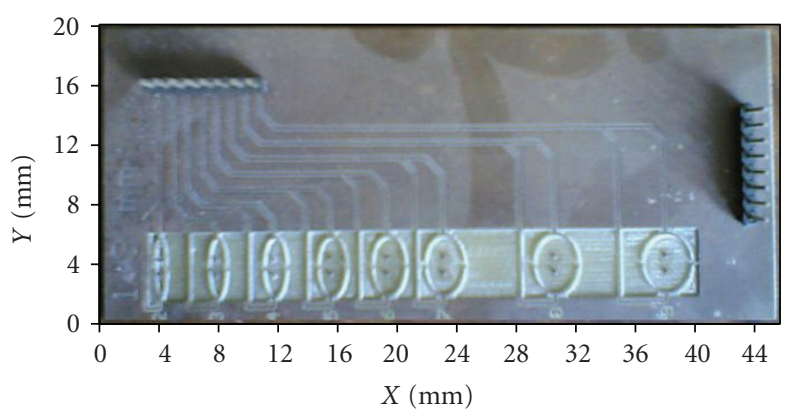

(a)

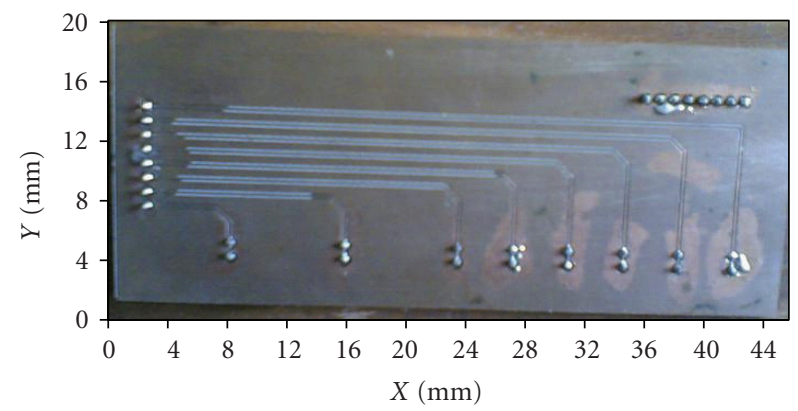

(b)

FIgURE 5: Photo of the fabricated electrode assembly of 8 electrodes, (a) top view showing the voltaic connections to the outer electrodes and (b) bottom (backside) view showing the voltaic connections to the inner electrodes. 


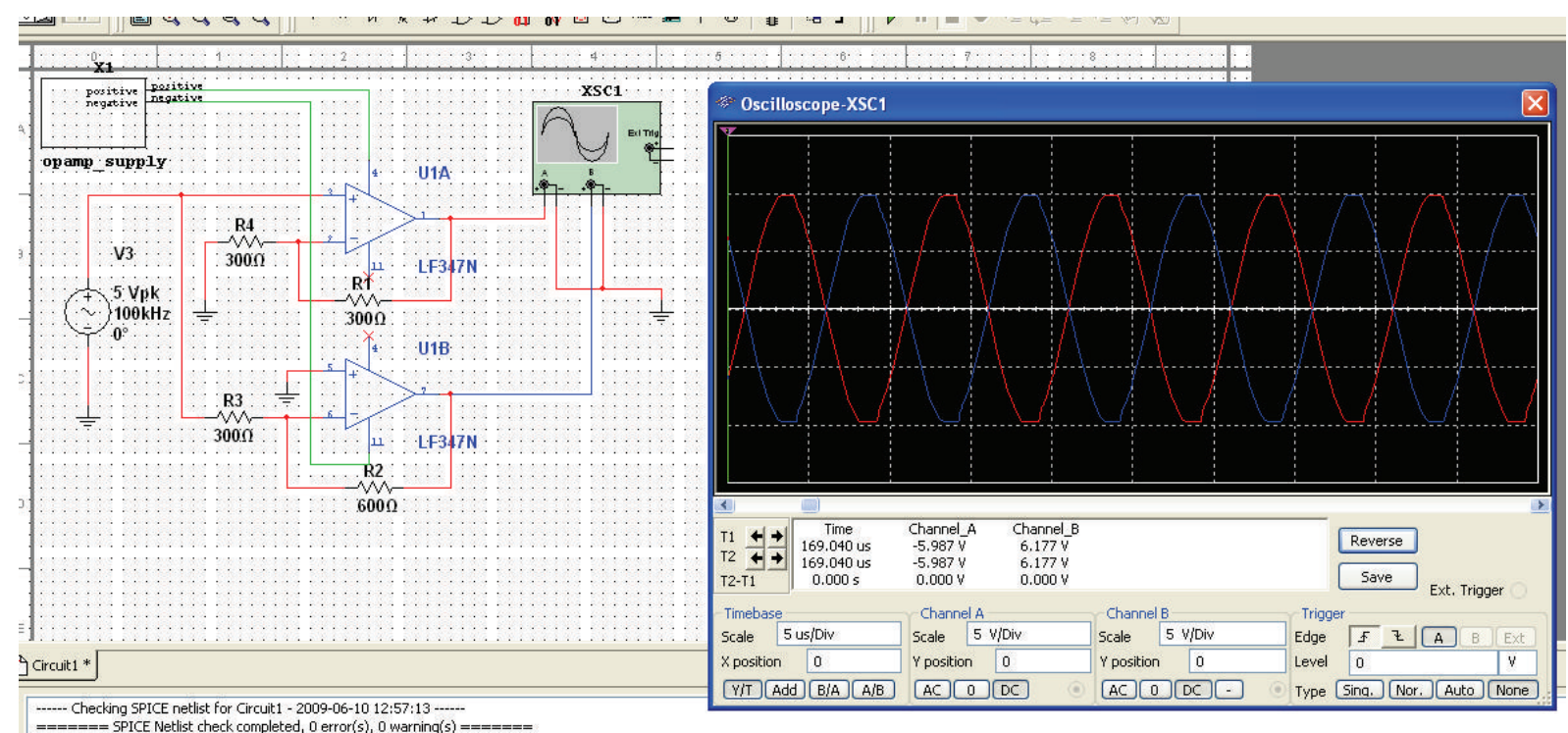

FIgURE 6: National Instrument Multisim simulation setup for electrode stimuli.

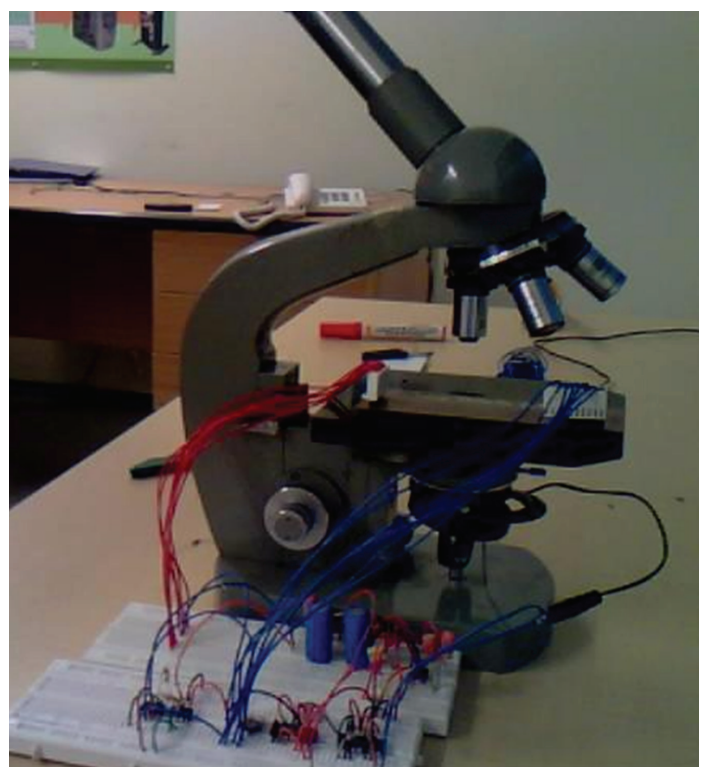

FIgure 7: Complete experimental setup with Olympus BX 51 research microscope for optical observation of nDEP using the electrode assembly.

satisfies the condition for negative DEP at a suitable crossover frequency based on the $K$-factor simulation in Figure 1 . At lower frequencies (near $1 \mathrm{kHz}$ ) copper would oxidize, thus the electrode assembly was washed with dilute sulphuric acid $\left(\mathrm{H}_{2} \mathrm{SO}_{4}\right)$ before each use. The electrode excitation voltage was varied between $5 \mathrm{~V}$ and $20 \mathrm{~V}$, and DEP actuation was observed above approximately $15 \mathrm{~V}$. Also, particle movement due to DEP force was observed above around $100 \mathrm{kHz}$. The experimental setup with electrode stimuli circuit on breadboard, electrode assembly, and Olympus BX 51 research microscope for optical observation is shown in Figure 7.
Each of the eight electrodes were tested separately one at a time. Figure 8 shows the collection/accumulation of polystyrene particles in a nDEP cage within an electrode of $Z=0.75$, when suspended in distilled water and excited with a stimulus frequency of $100 \mathrm{kHz}$ (approx). Although in these tests the fluid media was static, it is expected to produce similar results in a laminar flow. Only the set of 4 electrodes with $Z \geq 0.7$ produced the required results. The average polystyrene particle size used in the experiment was $45 \mu \mathrm{m}$. The polystyrene particles were procured from Sigma-Aldrich [20]. The experimental result is thus reasonably close to the crossover frequency for nDEP for $45 \mu \mathrm{m}$ particles as shown in the simulation in Figure 1. The device (electrode assembly) was unable to successfully trap yeast cells in the voltage and frequency test range of the experiment. Suspending media other than distilled water such as ethanol, mannitol, and so forth were also investigated in an effort to actuate $\mathrm{nDEP}$ movements on yeast cells. Like most biocells the permittivity and conductivity of yeast cell depends on frequency. In general, yeast cell is known to show negative DEP behavior in mannitol solution. However, the lack in DEP mobility of the yeast cells in this case was attributable mostly to the large dimensions of the electrode devices (built using $\mathrm{PCB}$ ) as compared to the size of yeast cells (diameter $=8-$ $10 \mu \mathrm{m}$ ). This can be explained based on (5), as for smaller particles, DEP force will depend considerably on further reduced device dimensions (low micrometer lengths for the elliptic electrode's semimajor and semiminor axis) so that the electric field intensity (and gradient) is increased considerably. Of the eight elliptic electrodes fabricated using PCB prototyping, the smallest one had foci interelectrode separation of $900 \mu \mathrm{m}$. For the manipulation of yeast cells electrode separation needed to be $200 \mu \mathrm{m}$ or less which was not implementable using PCB prototyping resolution. However, through microfluidic chip [24] fabrication using today's nanometric CMOS process technologies this can be achieved quite easily. 


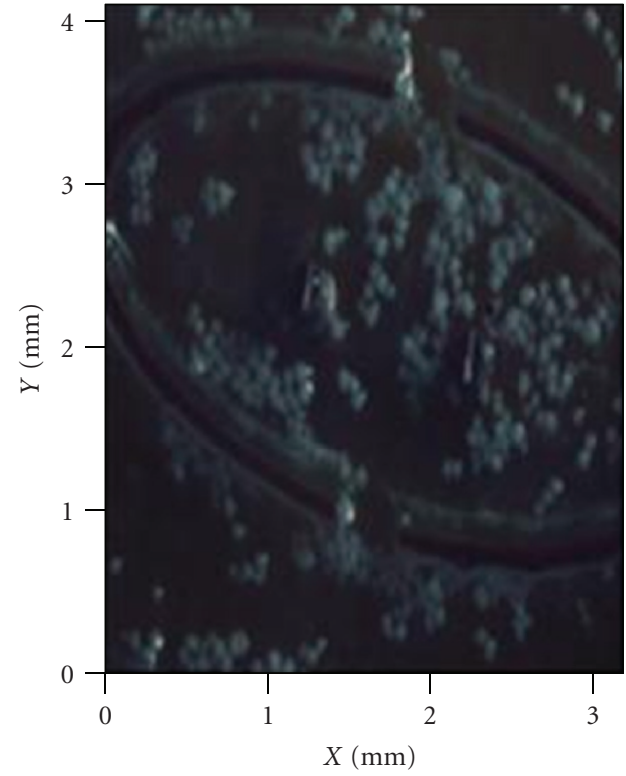

(a)

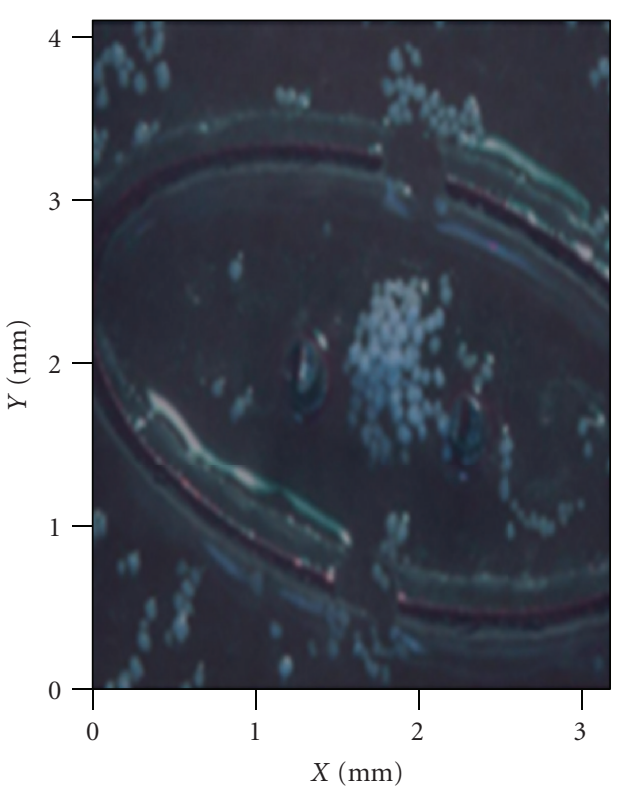

(b)

FIGURE 8: Microscopic view showing collection of polystyrene particles in a DEP cage using elliptic electrode, (a) in the absence of electric field and DEP force, (b) with negative DEP force in the presence of electric field.

\subsection{Advantages of the Proposed Elliptic Electrode Geometry.} There are quite a few advantages of using the proposed electrode geometry over available electrode designs. Large electric field gradients can be generated using a simple electrode set in elliptic geometry. The microchannels along the semiminor axis provides an easy way to remove the collected particles and also can act as inlet and outlet for laminar fluid flow. The actuation stimuli circuit is quite simple in design, and the electrodes can be easily integrated with capacitive sensing elements. It can be conveniently used to separate biomolecules based on size and dielectric properties [25]. Also, since there are two sets of electrodes, a combination of $\mathrm{nDEP}$ and $\mathrm{pDEP}$ can also be possibly used to remove parasitic traps in certain cases [26].

\section{Conclusion}

An investigation has been carried out on a set of PCB prototyped elliptic electrode assembly. The optimum geometry (semimajor to semiminor axis ratio) for negative DEP was determined through electric field simulations and verified experimentally using polystyrene particles suspended in distilled water. Also, the experimental negative DEP crossover frequency of around $100 \mathrm{kHz}$ for $45 \mu \mathrm{m}$ particles appear to agree closely with the simulations.

\section{Acknowledgments}

The authors would like to acknowledge assistance with experimental preparations from Dr. Uma Khurma and Professor Jagjit Khurma of the University of South Pacific, Mr. James McIntyre, Professor Ian Maddox, and Dr. Justin
O'Sullivan of Massey University Albany. Acknowledgment is also due to Mrs. Divya Dhawan of PEC. In addition, the quality of the paper was considerably enhanced by the reviewer's comments.

\section{References}

[1] G. Medoro, N. Manaresi, A. Leonardi, L. Altomare, M. Tartagni, and R. Guerrieri, "A lab-on-a-chip for cell detection and manipulation," IEEE Sensors Journal, vol. 3, no. 3, pp. 317325, 2003.

[2] Y. H. Ghallab and W. Badawy, "A novel cmos lab-on-a-chip for biomedical applications," in Proceedings of IEEE International Symposium on Circuits and Systems (ISCAS '05), pp. 13461349, May 2005.

[3] N. A. Campbell and J. B. Reece, Biology, Pearson, San Francisco, Calif, USA, 2005.

[4] U. Seger, S. Gawad, R. Johann, A. Bertsch, and P. Renaud, "Cell immersion and cell dipping in microfluidic devices," Lab on a Chip, vol. 4, no. 2, pp. 148-151, 2004.

[5] H. Morgan and N. G. Green, AC Electrokinetics: Colloids and Nano-Particles, Research Studies Press Ltd., Hertfordshire, UK, 2003.

[6] R. Pethig and G. H. Markx, "Applications of dielectrophoresis in biotechnology," Trends in Biotechnology, vol. 15, no. 10, pp. 426-432, 1997.

[7] H. Morgan, A. G. Izquierdo, D. Bakewell, N. G. Green, and A. Ramos, "The dielectrophoretic and travelling wave forces generated by interdigitated electrode arrays: analytical solution using Fourier series," Journal of Physics D, vol. 34, no. 10, pp. 1553-1561, 2001.

[8] W. M. Arnold and U. Zimmermann, "Rotation of an isolated cell in a rotating electric field," Naturwissenschaften, vol. 69, no. 6, pp. 297-298, 1982. 
[9] T. B. Jones and M. J. McCarthy, "Electrode geometries for dielectrophoretic levitation," Journal of Electrostatics, vol. 11, no. 2, pp. 71-83, 1981.

[10] J. T. Y. Lin and J. T. W. Yeow, "Enhancing dielectrophoresis effect through novel electrode geometry," Biomedical Microdevices, vol. 9, no. 6, pp. 823-831, 2007.

[11] K. Khoshmanesh, S. Nahavandi, S. Baratchi, A. Mitchell, and K. Kalantar-zadeh, "Dielectrophoretic platforms for biomicrofluidic systems," Biosensors and Bioelectronics, vol. 26, no. 5, pp. 1800-1814, 2011.

[12] G. Medoro, N. Manaresi, A. Leonardi, L. Altomare, M. Tartagni, and R. Guerrieri, "A lab-on-a-chip for cell detection and manipulation," IEEE Sensors Journal, vol. 3, no. 3, pp. 317325, 2003.

[13] IL. Doh and Y. H. Cho, "A continuous cell separation chip using hydrodynamic dielectrophoresis (DEP) process," Sensors and Actuators, A, vol. 121, no. 1, pp. 59-65, 2005.

[14] A. H. Pohl, Dielectrophoresis, Cambridge University Press, Cambridge, UK, 1978.

[15] H. A. Pohl, "Some effects of nonuniform fields on dielectrics," Journal of Applied Physics, vol. 29, no. 8, pp. 1182-1188, 1958.

[16] T. Jones, Electromechanics of Particles, Cambridge University Press, New York, NY, USA, 1995.

[17] T. B. Jones and G. A. Kallio, "Dielectrophoretic levitation of spheres and shells," Journal of Electrostatics, vol. 6, no. 3, pp. 207-224, 1979.

[18] W. M. Arnold, H. P. Schwan, and U. Zimmermann, "Surface conductance and other properties of latex particles measured by electrorotation," Journal of Physical Chemistry, vol. 91, no. 19, pp. 5093-5098, 1987.

[19] K. Khoshmanesh, C. Zhang, S. Nahavandi et al., "Size based separation of microparticles using a dielectrophoretic activated system," Journal of Applied Physics, vol. 108, no. 3, Article ID 034904, 7 pages, 2010.

[20] "Sigma-Aldrich," http://www.sigmaaldrich.com.

[21] C. M. White, L. A. Holland, and P. Famouri, "Application of capillary electrophoresis to predict crossover frequency of polystyrene particles in dielectrophoresis," Electrophoresis, vol. 31, no. 15, pp. 2664-2671, 2010.

[22] W. M. Arnold, "Positioning and levitation media for the separation of biological cells," IEEE Transactions on Industry Applications, vol. 37, no. 5, pp. 1468-1475, 2001.

[23] Y. Wang and M. N. Afsar, "Measurement of complex permittivity of liquid dielectrics," Microwave and Optical Technology Letters, vol. 34, no. 4, pp. 240-243, 2002.

[24] T. P. Hunt, D. Issadore, and R. M. Westervelt, "Integrated circuit/microfluidic chip to programmably trap and move cells and droplets with dielectrophoresis," Lab on a Chip, vol. 8, no. 1, pp. 81-87, 2007.

[25] S. Agastin, M. R. King, and T. B. Jones, "Rapid enrichment of biomolecules using simultaneous liquid and particulate dielectrophoresis," Lab on a chip, vol. 9, no. 16, pp. 2319-2325, 2009.

[26] M. Urdaneta and E. Smela, "Parasitic trap cancellation using multiple frequency dielectrophoresis, demonstrated by loading cells into cages," Lab on a Chip, vol. 8, no. 4, pp. 550$556,2008$. 

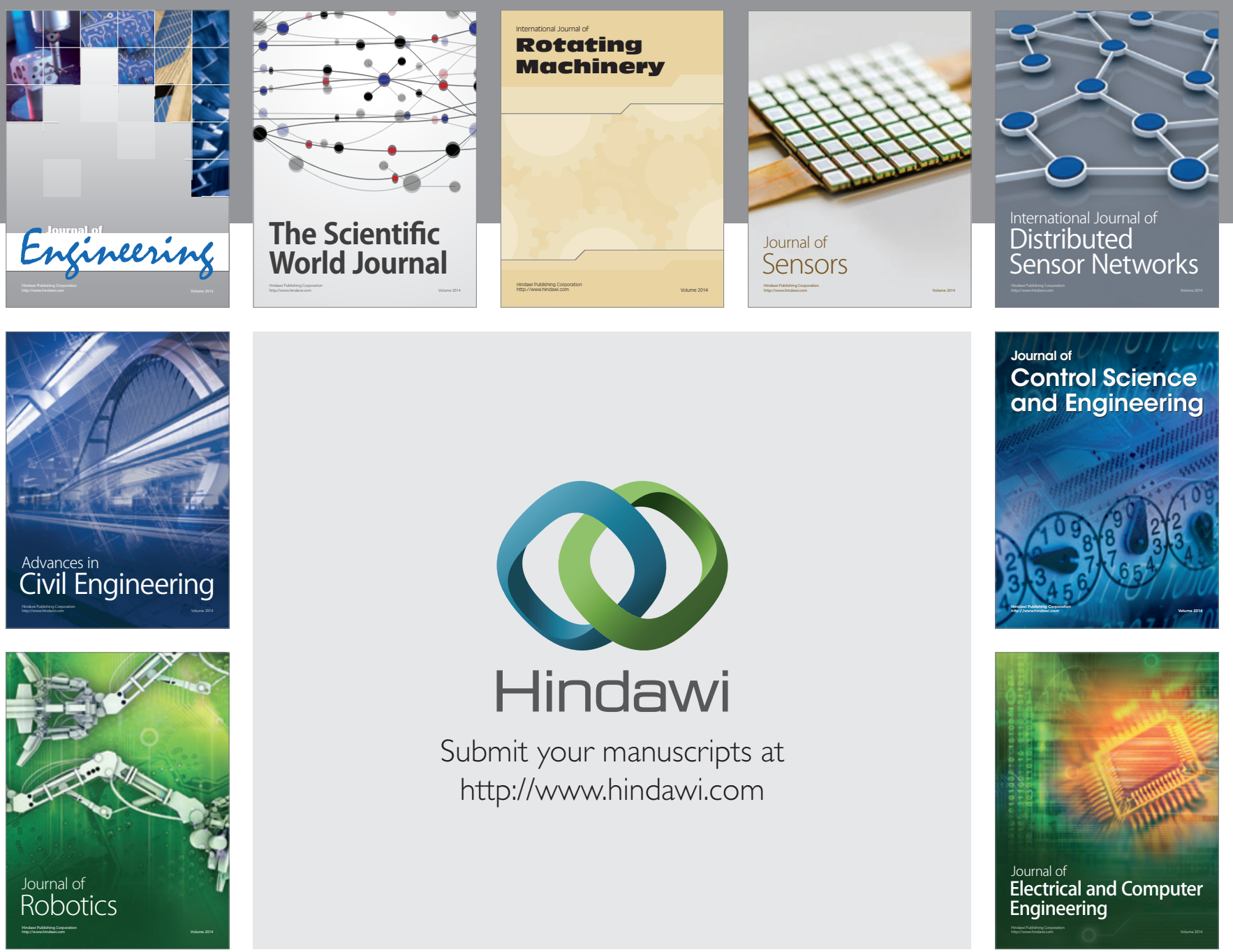

Submit your manuscripts at

http://www.hindawi.com
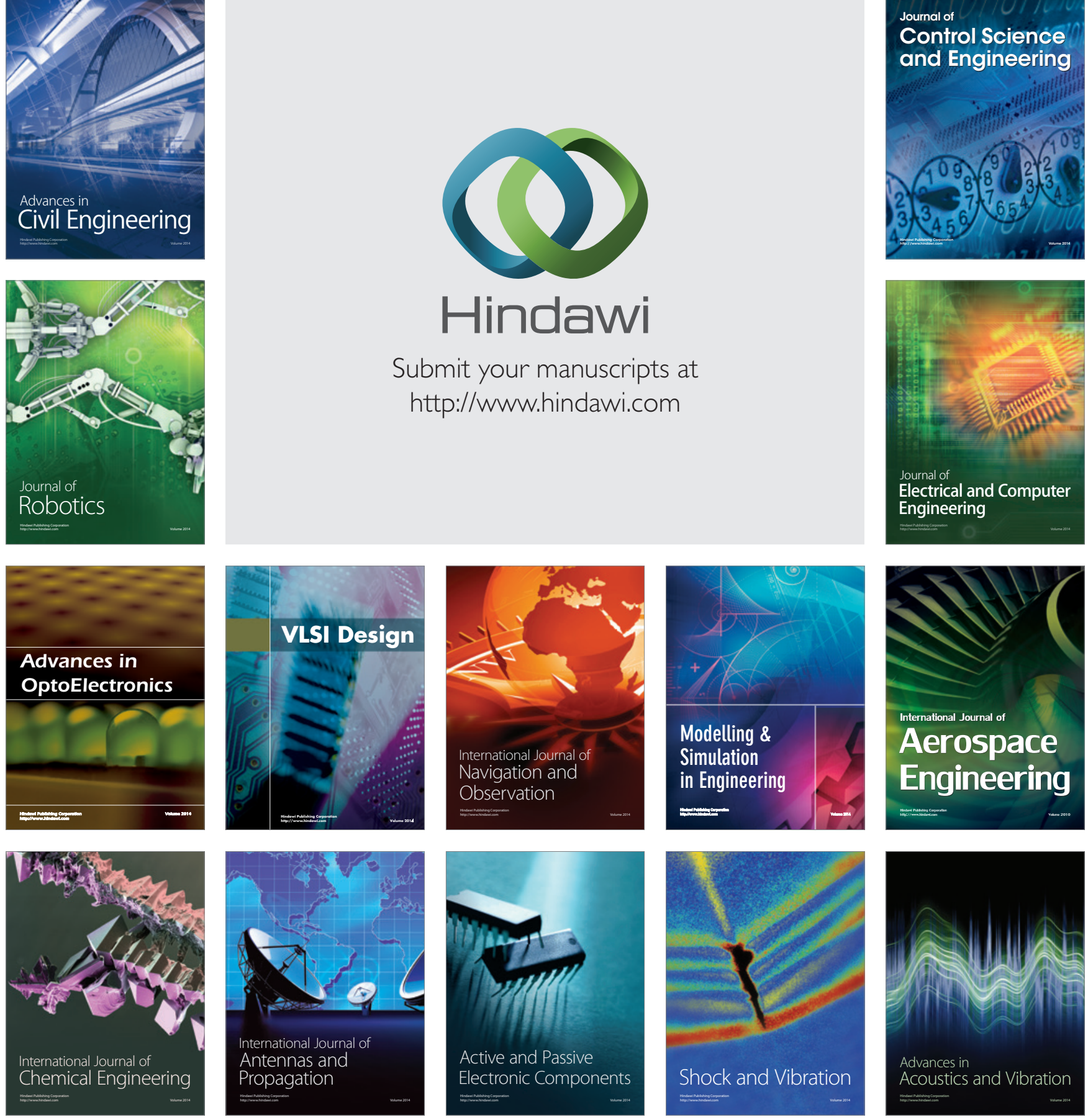